A0037: Comparing Hearing Outcomes in $\mathrm{CO}^{2}$ Laser Stapedotomies and Routine Stapedotomies

Thomas L., Lepcha A.

Background Hearing loss in otosclerosis is caused due to abnormal bone growth in the otic capsule. The current standard of care for surgical management of otosclerosis is small fenestra stapedotomy, which can be done using $\mathrm{CO}^{2}$ lasers or by conventional methods. In this case series, we assessed postoperative hearing outcomes in patients who underwent LASER stapedotomies and compared them to patients who underwent the classical method of stapedotomies.

Aim To assess whether laser stapedotomies are superior to classical stapedotomies in terms of hearing outcome.

Methods The medical charts of four patients who underwent laser stapedotomies were retrospectively reviewed and compared with four age-matched controls who underwent classical stapedotomies.

Results The postoperative air conduction thresholds and air-bone gaps at 250, 500, 1,000, 2,000, and 4,000 Hz were studied. There was found to be no statistically significant difference in the postoperative hearing between the two groups.

Conclusion There appears to be no significant difference in the postoperative hearing outcomes irrespective of the surgical method used.

Clinical Significance Otosclerosis is one of the causes for conductive hearing loss in several Indian patients. The use of CO2 laser has been rising recently owing to its no touch principle and high precision. However, the cost for the patient as well as the hospital has increased greatly by the use of lasers. As the main outcome for a successful stapedotomy is improvement in hearing, the decision of which method to use should be based on clinical factors and financial ability of the patients.

\section{A0038: Case Report: Herpes Zoster Cephalicus in an Immunocompromised Patient \\ Nalina}

Introduction Ear-nose-throat-head and neck manifestations due to HIV infection are common in otolaryngological practice. We would like to present a case of herpes zoster cephalicus in a middle-aged lady who was HIV positive.

Case Presentation A 38-year-old lady presented to us with complete weakness of left side of the face, otalgia, nasal regurgitation of fluids during swallowing and dysphagia. ENT examination revealed dried hemorrhagic crusts along the sensory distribution of the facial nerve in the left ear with left-sided lower motor neuron type of facial paralysis, House-Brackmann grade IV. A horizontal paralytic type of nystagmus to the right was present. Throat examination revealed ipsilateral paralysis of the soft palate. Laryngo-endoscopy revealed left-sided vocal cord palsy. A clinical diagnosis of herpes zoster cephalicus was made and treatment was started with oral acyclovir and prednisolone after consultation with an internist. The patient made a partial recovery.
Discussion Herpes zoster cephalicus is an uncommon clinical syndrome seen in immunocompromised patients which occurs due to reactivation of herpes zoster virus dormant in the involved cranial nerve ganglia of head and neck. Patients present to us with acute onset of facial nerve palsy with otalgia with vesicles seen in the sensory distribution of the facial nerve, absence of pharyngeal reflexes, paralysis of palate, and vocal cord. Some patients may also have labyrinthine involvement. Then, patients should be aggressively treated with acyclovir and steroids along with antiretroviral therapy.

Conclusion Immunocompromised patients having multiple lower cranial nerve palsies of head and neck should be suspected to have herpes zoster cephalicus. These patients should be aggressively treated to prevent unwanted permanent motor deficits.

\section{A0039: Ear Lobe Repair: An Innovative Method \\ Nikisha G. N., Madana Gopal}

Introduction Ear piercing and ornament wearing is a historical tradition. Dilated ear lobe and torn earlobe usually results from sudden trauma or continuous use of heavy earrings. Various surgical techniques have been described for the correction of the same. Each procedure has its own pros and cons. The choice will depend on individual preferences and the defect, partial or total, and should offer a lower chance of recurrence. The corrections can be made with or without preservation of the earring orifice. This case report explains a novel technique to repair ear lobe using tissue fibrin.

Case Presentation A 67-year-old female presented with torn ear lobe due to usage of heavy ornaments. She was not a diabetic or hypertensive. All routine investigations were found to be normal. Ear lobe repair was done for her using tissue fibrin sealant glue. Postoperatively she was followed-up. A good postoperative result was achieved. Ear lobe sutured skin healed without any complications. Postoperative course was short and pain was reduced.

Discussion Cleft ear lobe and torn ear lobe have been known since ancient times. Egyptian pharaoh Tutankhamen was one of the earliest known to have stretched ear lobe. Many techniques have been described to repair ear lobe. The clinical usage of fibrin sealant has been extensively studied for diverse applications including tissue adhesion, wound closure, hemostasis, closure of cerebrospinal fluid (CSF) leaks, vascular embolization, and application of skin grafts. The tissue glue usually slough-off with wound reepithelialization within 5 to 10 days and do not require removal. The use of fibrin sealant for ear lobe repair gives good result, fast healing, good patient compliance, and good cosmetic result.

Conclusion The use of tissue fibrin sealant has several advantages over conventional sutures like their fast and painless application, rapid setting which reduce the total operating time and their antibacterial properties. It also has a good cosmetic result. 